(G) A basic difference in performance between GS and MGS is that once GS loses orthogonality, it produces almost identical vectors thereafter. The maximum inner product is usually .999 etc. (and this is to be expected from the error analysis of 3 ). On the other hand, MGS continues to generate distinct, if not orthogonal, vectors. Thus the inner products obtained (after orthogonality is lost) normally range between 0.1 and 0.9 . This is no doubt due to the fact that $v_{k}$ is always orthogonal to $v_{k-1}$ within machine accuracy. This is suggested by the error analysis and confirmed by experiment.

I wish to acknowledge the able assistance of Rex Wolf in preparing the programs for these experiments.

1. P. J. Davis \& P. RABINowitz, "Advances in orthonormalizing computation," Advances in Computers, F. L. Alt (Ed.), Vol. 2, Academic Press, New York, 1961, pp. 55-133. MR 25 * 1636 .

\title{
A Matrix Reduction Problem
}

\section{By J. W. Moon and L. Moser}

1. Introduction. Let $A_{n}$ denote an $n$ by $n$ matrix of 0 's and 1's that is nonsingular over the field of residues modulo 2. Fine and Niven [1] have shown that these are $c_{n} 2^{n^{2}}$ such matrices where.

$$
c_{n}=\prod_{k=1}^{n}\left(1-\left(\frac{1}{2}\right)^{k}\right) .
$$

Let $f\left(A_{n}\right)$ denote the minimum number of operations needed to transform $A_{n}$ into the identity matrix $I_{n}$. (It may be necessary, of course, to interchange certain rows but we do not count this as an operation.) The object in this note is to give bounds for $f\left(A_{n}\right)$ which at least determine its order of magnitude for almost all matrices $A_{n}$. These may be of some interest in connection with the question of the minimal number of operations required to invert a matrix. Indeed our methods and results apply with only minor modifications to the case of matrices with real elements provided that in performing arithmetic operations only a fixed number of significant digits is retained.

Theorem. There exist positive constants $c_{1}$ and $c_{2}$ such that

$$
\frac{c_{1} n^{2}}{\log n}<f\left(A_{n}\right)<\frac{c_{2} n^{2}}{\log n}
$$

for almost all matrices $A_{n}$; i.e. for all but a fraction which tends to zero as $n$ tends to infinity.

2. A Lower Bound for $f\left(A_{n}\right)$. We will show that

$$
f\left(A_{n}\right)>\lambda=\frac{\left(\frac{1}{2}-\epsilon\right) n^{2}}{\log _{2} n}
$$

for almost all matrices $A_{n}$ where $\epsilon$ is an arbitrary positive constant.

Received July 19, 1965. Revised October 1, 1965. 
If $A_{n}$ can be transformed into $I_{n}$ in $t$ operations, then clearly $I_{n}$ can also be transformed into $A_{n}$ in $t$ operations. Hence, the number of matrices $A_{n}$ such that $f\left(A_{n}\right) \leqq$ $\lambda$ certainly does not exceed

$$
\left(3\left(\begin{array}{l}
n \\
2
\end{array}\right)\right)^{\lambda} n !
$$

since after choosing a pair of rows in one of $\left(\begin{array}{l}n \\ 2\end{array}\right)$ ways there are 3 alternatives; we may add either of the rows to the other or we may leave them unchanged. The $n$ ! takes into account the number of ways of permuting the rows. But,

$$
\left(3\left(\begin{array}{l}
n \\
2
\end{array}\right)\right)^{\lambda} n !<\left(\frac{3}{2}\right)^{\lambda} n^{n+2 \lambda}
$$

The quotient of the last expression divided by $c_{n} 2^{n^{2}}$, the total number of matrices $A_{n}$, tends to zero. This suffices to complete the proof of the lower bound for $f\left(A_{n}\right)$.

3. An Upper Bound for $f\left(A_{n}\right)$. We will now show that if the matrix $A_{n}$ can be transformed into $I_{n}$ in a finite number of operations then it can be so transformed in at most $(4+\epsilon) n^{2} / \log _{2} n$ operations if $n>n(\epsilon)$.

Let $k$ be a positive integer less than $n$. We may suppose that the first row of $A_{n}$ does not begin with $k$ 0's. Add the first row to every other row that begins with the same $k$ entries. Now consider the second row of the matrix that does not begin with $k$ 0's; add this row to every other row that begins with the same $k$ entries. Repeat this process as long as possible. We may then rearrange the rows to obtain a matrix in which no two of the first $t$ rows have the same first $k$ entries and the last $n-t$ rows all begin with $k 0$ 's, where $t$ is some integer not exceeding $2^{k}-1$. This has been accomplished with at most $n-t$ operations. It must be that $k \leqq t$ for otherwise it would be impossible to transform $A_{n}$ into $I_{n}$. At most $k(t-1)$ more operations are needed to change the first $k$ columns to upper triangular form with 1 's on the main diagonal. At the end of this stage at most $(n-t)+k(t-1) \leqq$ $n-3 k+k 2^{k}$ operations have been employed.

We now repeat this process to the entries in the next $k$ columns and bottom $n-k$ rows. With at most $n-3 k+k 2^{k}$ more operations we obtain a matrix which has 1's on the main diagonal and 0's below the main diagonal in the first $2 k$ rows. (We ignore the effect of these operations on the entries above the main diagonal for the time being.) By repeating this process at most $n / k+1$ times we will obtain an upper triangular matrix with ones down the main diagonal. It is clear that the operations performed at each stage do not affect the properties established at the preceding stage.

To obtain 0's above the main diagonal also, we now repeat this process, starting at the lower right corner and working up the main diagonal. Therefore, the number of operations necessary to transform $A_{n}$ into $I_{n}$, if it can be so transformed, does not exceed

$$
2\left(\frac{n}{k}+1\right)\left(n-3 k+k 2^{k}\right)=2\left\{n\left(\frac{n}{k}+2^{k}\right)+k\left(2^{k}-3\right)-2 n\right\} .
$$

If we now let $k=\left[\log _{2} n-\log _{2} \log _{2} n\right]$, then an upper bound for the resulting ex- 
pression is $4 n^{2} /\left(\log _{2} n-\log _{2} \log _{2} n-1\right)$. The result stated at the beginning of this section now follows immediately.

4. Generalizations. The corresponding problem may be considered for $n$ by $n$ matrices whose entries are taken from the integers $0,1, \cdots, k-1$. If an operation on such a matrix consists of adding a multiple of some row to some other row modulo $k$, then it can be shown that the foregoing theorem remains valid in this more general situation for any fixed value of $k$. In fact, the bounds in Sections 2 and 3 will still hold if $\log _{2} n$ is replaced by $\log _{k} n$.

1. N. J. Fine \& I. Niven, "The probability that a determinant be congruent to a $(\bmod m), "$ Bull. Amer. Math. Soc., v. 50, 1944, pp. 89-93.

\section{Evaluation of $I_{n}(b)=2 \pi^{-1} \int_{0}^{\infty}\left(\frac{\sin x}{x}\right)^{n} \cos (b x) d x$ and of \\ Similar Integrals}

\section{By Rory Thompson}

Medhurst and Roberts [1] suggest the problem of evaluating $I_{n}(b)$ for nonintegral values of $b$. There will be developed in this note an effective recursion scheme for such a calculation. In particular, it can be used to evaluate $I_{n}(0)$ for moderate values of $n$.

Following a suggestion by Hamming [2, p. 164], we differentiate $I_{n}(b)$ with respect to the parameter $b$, which is permissible by virtue of uniform convergence of the resulting integral for $n>2$ and continuity of the corresponding integrand with respect to both $x$ and $b$.

Thus we obtain

$$
\begin{aligned}
I_{n}{ }^{\prime}(b) & =-2 \pi^{-1} \int_{0}^{\infty}\left(\frac{\sin x}{x}\right)^{n-1} \sin x \sin (b x) d x \\
& =\pi^{-1} \int_{0}^{\infty}\left(\frac{\sin x}{x}\right)^{n-1}[\cos (b+1) x-\cos (b-1) x] d x \\
& =\frac{1}{2}\left[I_{n-1}(b+1)-I_{n-1}(b-1)\right] .
\end{aligned}
$$

If the first expression for $I_{n}{ }^{\prime}(b)$ is integrated by parts there results the relation

$$
\begin{aligned}
I_{n}{ }^{\prime}(b)= & (n-1) b^{-1} 2 \pi^{-1} \int_{0}^{\infty}\left(\frac{\sin x}{x}\right)^{n} \cos (b x) d x \\
& -n b^{-1} 2 \pi^{-1} \int_{0}^{\infty}\left(\frac{\sin x}{x}\right)^{n-1} \cos x \cos (b x) d x \\
& =\frac{n-1}{b} I_{n}(b)-\frac{n}{2 b}\left[I_{n-1}(b+1)+I_{n-1}(b-1)\right] .
\end{aligned}
$$

Received June 14, 1965. Revised August 19, 1965. 